\section{FRI0453 HOSPITALISATION AND SURVIVAL ANALYSIS IN SYSTEMIC SCLEROSIS PATIENTS WITH CONCOMITANT OR ISOLATED PULMONARY HYPERTENSION AND INTERSTITIAL LUNG DISEASE IN THE MULTIETHNIC SCLERODERMA COHORT SINGAPORE}

M. Noviani ${ }^{1}$, E.S. Seyed ${ }^{2}$, S.M.Y. Kua ${ }^{1}$, G.Y.L. Chan ${ }^{3}$, G.G. Teng ${ }^{4}$, W.G. Law ${ }^{3}$, A. Santosa ${ }^{4}$, A. Lim ${ }^{4}$, J.L. $\operatorname{Tan}^{5}$, E.L.W. Tay ${ }^{6}$, J.W.L. Yip ${ }^{6}$, S.C. Ng ${ }^{1}$, A.H.L. Low ${ }^{1}$ ${ }^{1}$ Department of Rheumatology and Immunology, Singapore General Hospital;

${ }^{2}$ Center for Quantitative Medicine, Duke-NUS Medical School; ${ }^{3}$ Department of Rheumatology, Allergy and Immunology, Tan Tock Seng Hospital; ${ }^{4}$ Department of Rheumatology, National University Health System; ${ }^{5}$ Department of Cardiology, National Heart Center Singapore; ${ }^{6}$ Department of Cardiology, National University Heart Center, Singapore, Singapore

Background: Concomitant pulmonary hypertension and interstitial lung disease in systemic sclerosis (SSc-PH-ILD) represents a distinct subpopulation of SSc with poorer prognosis in Western studies. In Asian patients, characterisation of SSc-PH-ILD is still lacking.

Objectives: To analyse hospital admissions, survival and prognostic markers among SSc patients with PH, ILD or concomitant PH-ILD in the Scleroderma Cohort Singapore.

Methods: In this study involving 3 tertiary Rheumatology institutions Jan 2008 to, Oct 2016 SSc patients with significant pulmonary involvement were included. ILD was based on high resolution computed tomography and predicted FVC $<70 \%$. $\mathrm{PH}$ was based on either echocardiographic systolic pulmonary arterial pressure (sPAP) $\geq 50 \mathrm{mmHg}$, or right heart catheterization (RHC) findings of mean $\mathrm{PAP}>25 \mathrm{mmHg}$. Hospitalisation rates and survival of SSc patients with $\mathrm{PH}$, ILD or PH-ILD were compared. Risk factors of poor outcomes were identified by multivariate stepwise Cox regression analysis.

Results: Among 490 patients, 92 had ILD, $50 \mathrm{PH}$ and $43 \mathrm{PH}$-ILD (table 1). Of 93 patients with $\mathrm{PH}$ or $\mathrm{PH}-\mathrm{ILD}, 56$ were based on echocardiography and 37 on RHC. The 5 year survival was $79 \%, 87 \%$ and $90 \%$ in PH, PH-ILD and ILD subgroup, respectively (figure 1). In multivariable analysis, $\mathrm{PH}$ was significantly associated with 2.8-fold increased risk of death. Male gender, malabsorption, digital ulcerations and renal crisis were also significantly associated with mortality (table 2). No significant difference in hospital admissions/year among different subgroups. Increased hospital admissions were associated with renal crisis, right heart failure and use of $\mathrm{PH}$ medications.

Abstract FRI0453 - Table 1. Clinical characteristics

\begin{tabular}{|c|c|c|c|c|}
\hline & $\mathrm{PH}(\mathrm{n}=50)$ & ILD $(n=92)$ & $\begin{array}{l}\text { PH-ILD } \\
(n=43)\end{array}$ & $\begin{array}{l}\text { No } \mathrm{PH} / \mathrm{ILD} \\
(\mathrm{n}=305)\end{array}$ \\
\hline Female, $\mathrm{n}$ & 44 & 76 & 38 & 270 \\
\hline Follow up duration (months & 53.46 & 101.5 & $88.71 \pm 65.53$ & $64.66 \pm 36.27$ \\
\hline$\pm \mathrm{SD})$ & \pm 55.76 & \pm 80.04 & & \\
\hline Age at SSc diagnosis (years & 51.08 & 46.87 & $53.84 \pm 15.17$ & $46.44 \pm 14.59$ \\
\hline$\pm \mathrm{SD})$ & \pm 16.44 & \pm 12.4 & & \\
\hline $\begin{array}{l}\text { Duration of SSc at entry (years } \\
\pm S D \text { ) }\end{array}$ & $5.85 \pm 6.95$ & $6.93 \pm 7.45$ & $6.14 \pm 7.98$ & $5.20 \pm 8.24$ \\
\hline Dc-SSc, n & 13 & 45 & 11 & 100 \\
\hline $\mathrm{PH}$ specific treatments ${ }^{+}, \mathrm{n}$ & 28 & $\mathrm{~N} / \mathrm{A}$ & 26 & $\mathrm{~N} / \mathrm{A}$ \\
\hline Immunosuppressants ${ }^{++}, \mathrm{n}$ & 25 & 64 & 27 & 176 \\
\hline
\end{tabular}

+Prostacyclin, phosphodiesterase type 5 inhibitors, endothelin receptor antagonist

$+{ }^{+}$Methotrexate, cyclophosphamide, mycophenolate mofetil.

Abstract FRI0453 - Table 2. Survival analysis

\begin{tabular}{lcc}
\hline & Hazard Ratio $(95 \% \mathrm{Cl})$ & $\mathrm{P}$ Value \\
\hline Male gender & $2.85(1.53-5.33)$ & 0.0010 \\
Malabsorption & $2.89(1.67-5.01)$ & 0.0002 \\
Renal crisis & $2.00(1.00-3.99)$ & 0.0490 \\
Digital ulcerations & $2.06(1.21-3.50)$ & 0.0076 \\
\hline
\end{tabular}

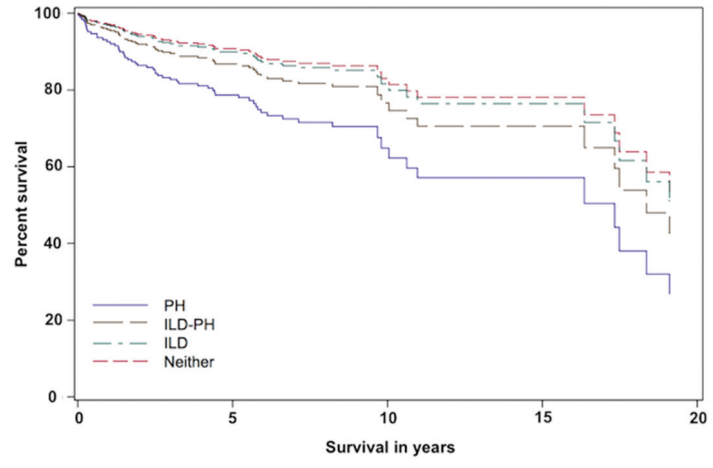

Abstract FRI0453 - Figure 1. Adjusted survival curve comparing survival of SSc patients with $\mathrm{PH}$, ILD, and concomittant PH-ILD. X-axis shows years of survival from diagnosis of $\mathrm{PH}$ or ILD.

Conclusions: Compared to those with ILD or PH-ILD, SSc-PH patients had increased mortality, but not hospitalisation rates. This could be due to small sample size or short follow up duration. We identified risk factors associated with worse outcomes in SSc patients with significant pulmonary involvement.

Disclosure of Interest: None declared

DOI: 10.1136/annrheumdis-2018-eular.3185

\section{FRI0454 ARTERIAL STIFFNESS OF THE FOREARM IS ASSOCIATED WITH NAIL-FOLD CAPILLARY COUNT IN SSC: A NOVEL MARKER OF EARLY VASCULOPATHY?}

M.M. Habing ${ }^{1}$, A. Eman Abdulle ${ }^{1}$, A.M. van Roon ${ }^{1}$, A.M. van Roon ${ }^{1}$, H. van Goor ${ }^{2}$, H. Bootsma ${ }^{3}$, A.J. Smit ${ }^{1}$, D.J. Mulder ${ }^{1}{ }^{1}$ Dept. of Internal medicine, div. Vascular Medicine; ${ }^{2}$ Dept. of Pathology and Medical Biology, Section Pathology; ${ }^{3}$ Dept. of Rheumatology and Clinical Immunology, University of Groningen - University Medical Centre Groningen., Groningen, Netherlands

Background: Microvascular disease, characterised by rarefaction of capillaries, is the hallmark of systemic sclerosis. Remarkably, obliteration of the ulnar and radial artery is regularly observed, implicating involvement of the larger forearm arteries. Pulse wave velocity (PWV) is a widely accepted non-invasive measure for arterial stiffness and may serve as an early biomarker of forearm artery involve ment, before the occurrence of irreversible arterial obliteration.

Objectives: The aim of the current study was to investigate arterial stiffness of the aorta and of the upper extremities in SSc patients compared to healthy con trols and to correlate these findings with nail-fold capillary count, skin involvement, and extent of disease.

Methods: In total, 19 SSc patients (median age 51 years, 68\% female) and 19 age and gender matched healthy controls (median age 53 years, 68\% female) were included. Patients characteristics were obtained and blood was drawn. Measurements of arterial stiffness were carried out by using the SphygmoCor System (AtCor Medical, Sydney, Australia) and pressure waveforms were meas ured at four sites, i.e. carotid, femoral, brachial, and radial. Aortic PWV was defined as carotid-femoral (cf) PWV. Upper extremity PWV was measured as carotid-brachial (cb) and carotid-radial (cr) PWV, and the ratio between cbPWV/ crPWV was used as an indication of the relative change in PWV in the forearm. Capillary count was defined as the mean capillary count per $3 \mathrm{~mm}$ of 8 fingers. Skin involvement was assessed by the modified rodnan skin score. The number of ACR/EULAR 2013 criteria points was used as a surrogate for extent of disease. Results: Upper extremity PWV measures were significantly higher compared to aortic PWV in patients and in controls (SSc: $p<0.001 ; \mathrm{HC}: \mathrm{p}=0.03$ ), but did not significantly differ between both groups (table 1). CbPWV/crPWV ratio correlated strongly with capillary count $(r=-0.55, p=0.022$, figure 1$)$ in SSc patients with a borderline significant trend in regards to its relation with the extent of disease $(r=0.48, p=0.053)$ and skin involvement $(r=0.41, p=0.10)$. 